\title{
A Direct Algorithm for Pole Placement by State-derivative Feedback for Single-input Linear Systems
}

\author{
Taha H. S. Abdelaziz, M. Valášek
}

This paper deals with the direct solution of the pole placement problem for single-input linear systems using state-derivative feedback. This pole placement problem is always solvable for any controllable systems if all eigenvalues of the original system are nonzero. Then any arbitrary closed-loop poles can be placed in order to achieve the desired system performance. The solving procedure results in a formula similar to the Ackermann formula. Its derivation is based on the transformation of a linear single-input system into Frobenius canonical form by a special coordinate transformation, then solving the pole placement problem by state derivative feedback. Finally the solution is extended also for single-input time-varying control systems. The simulation results are included to show the effectiveness of the proposed approach.

Keywords: pole placement, state-derivative feedback, linear single-input systems, feedback stabilization.

\section{Introduction}

An important problem in the theory and practice of control system design is the design of feedback controllers, which place the closed-loop poles of a linear system at desired locations. The literature in this field is quite rich. The state feedback control problem has been given a lot of attention in the control community during the last three decades. Several researchers have developed design methods for a wide class of linear systems under full-state feedback with the objective of stabilizing control systems (e.g. [1-10]). In designing control systems based on pole placement, it may be satisfactory in practice that the closed-loop system has all poles at a desired location.

However, this paper focuses on a special feedback using only state derivatives instead of full state feedback. Therefore this feedback is called state derivative feedback. The problem of arbitrary pole placement using full state derivative feedback naturally arises. To the best of the authors' knowledge, there has not yet been any general study solving this feedback for pole placement.

The motivation for the state derivative feedback comes from controlled vibration suppression of mechanical systems. The main sensors of vibration are accelerometers. From accelerations it is possible to reconstruct velocities with reasonable accuracy, but not the displacements. Therefore the available signals for feedback are accelerations and velocities only, and these are exactly the derivatives of the states of mechanical systems which are the velocities and displacements. One necessary condition for a control strategy to be implementable is that it must use the available measured responses to determine the control action. All of the previous research in the control has assumed that all of the states can be directly measured (i.e., full state feedback).

To control this class of systems, many papers have been published (e.g. [11-16]) describing the acceleration feedback for controlled vibration suppression. However, the pole placement approach for feedback gain determination has not been used at all or has not been solved generally. The approach in [11-14] is based on dynamic derivative output feedback. The feedback uses acceleration only (the velocity is not used, therefore it is not full state derivative feedback, but only output derivative feedback) and the acceleration is processed by a dynamic filter (dynamic feedback). The feedback gains are determined using root locus analysis [11-15], optimization of the $\mathrm{H}_{2}$ norm of the closed loop transfer function [14] or using just numerical parameter optimization of performance indices [16]. Other papers dealing with acceleration feedback for mechanical systems are [17-18], but here the feedback uses all states (positions, velocities) and accelerations additionally. Recently, paper [19] has presented a nonlinear controller based on the state-derivative feedback control for a magnetic bearing. The state-derivative feedback is used for feedback linearization and not for pole placement.

In this paper the problem of pole placement by state derivative feedback for single-input linear systems, both time invariant and time varying, is generally formulated and solved. The solution is based on recent efficient techniques for solving the pole placement problem by state feedback for SISO and MIMO linear time-invariant and time-varying systems $[8,9,10]$. It uses the transformation of a linear system into Frobenius canonical form and results in different versions of Ackermann's formula. This methodology is also utilized in this paper.

In summary, this paper is organized as follows. In section 2 , we begin with the general formulation of the problem followed by the solution for single-input linear time invariant systems. Section 3 deals with the extension of this pole placement for time-varying systems. In section 4 , the illustrative examples and simulation results for several systems are presented. Finally, conclusions follow in section 5.

\section{Pole placement by state-derivative feedback for linear time-invariant systems}

Pole placement by state-derivative feedback is solved in this section for linear time-invariant systems using the technique from $[8,9,10]$. 


\subsection{Pole placement problem formulation}

Consider a controllable linear time-invariant single-input system,

$$
\dot{x}(t)=\boldsymbol{A} x(t)+\boldsymbol{B} u(t), \quad x\left(t_{0}\right)=x_{\mathbf{0}},
$$

where $x(t) \in \mathrm{R}^{n}$ and $u(t) \in \mathrm{R}$ are the state vector and the scalar control input, respectively, while $\boldsymbol{A} \in \mathrm{R}^{n \times n}$ and $\boldsymbol{B} \in \mathrm{R}^{n}$ are the system matrix and control gain vector, respectively. The characteristic polynomial of matrix $A$ can be given by $\operatorname{det}[\mathrm{s} \boldsymbol{I}-\boldsymbol{A}]=\mathrm{s}^{n}+a_{n-1} \mathrm{~s}^{n-1}+\Lambda+a_{1} \mathrm{~s}+a_{0}=0$,

where $\boldsymbol{a}=\left[a_{0}, a_{1}, \ldots, a_{n-1}\right]$ are the coefficients of the characteristic polynomial and $\boldsymbol{I} \in \mathrm{R}^{n \times n}$ is the identity matrix. It is known that $a_{0}=\operatorname{det}(-\boldsymbol{A})=(-1)^{n} \operatorname{det}(\boldsymbol{A})$ and $a_{n-1}=-\operatorname{trace}(A)$.

The objective is to place the desired poles for the closed-loop system using the constant state-derivative feedback control

$$
u=-\boldsymbol{K} \dot{\boldsymbol{x}},
$$

that enforces a desired characteristic behavior for the states and thus stabilizes the system. Using the feedback (3) the closed-loop system becomes

$$
\dot{\boldsymbol{x}}(t)=[\boldsymbol{I}+\boldsymbol{B} \boldsymbol{K}]^{-1} \boldsymbol{A} \boldsymbol{x},
$$

The design problem is to find the feedback gain matrix $\boldsymbol{K}$ such that the closed-loop poles, $\left\{\lambda_{1}, \lambda_{2}, \ldots, \lambda_{n}\right\}$ satisfying $\operatorname{spec}(A) \cap\left\{\lambda_{1}, \lambda_{2}, \ldots, \lambda_{n}\right\}=0$, of system (4) are assigned at the desired values. A solution of this problem using the procedures of state feedback is difficult, and a direct approach usually leads to iterative optimization. The solution to this problem is further accomplished by utilizing a transformation of this system to the Frobenius canonical form and placing the desired eigenvalues.

\subsection{Transformation into Frobenius canonical form for time-invariant systems}

Frobenius canonical form is constructed by transforming the state vector to a new coordinate system in which the system equation takes a particular form. Let us take the following time-invariant state transformation,

$$
z(t)=Q^{-1} x(t),
$$

where $z(t) \in \mathrm{R}^{n}$ is the new (transformed) state variable vector and $\boldsymbol{Q}^{-1} \in \mathrm{R}^{n \times n}$ is the transformation matrix. The original system is transformed into a system with the transformed system matrix $\boldsymbol{A}_{F} \in \mathrm{R}^{n \times n}$ and the transformed control gain vector $\boldsymbol{B}_{F} \in \mathrm{R}^{n}$, which are given by

$$
\boldsymbol{A}_{F}=\boldsymbol{Q}^{-1} \boldsymbol{A} \boldsymbol{Q}, \quad \boldsymbol{B}_{F}=\boldsymbol{Q}^{-1} \boldsymbol{B} .
$$

If the transformation matrix is chosen as

$$
Q^{-1}=\left(\begin{array}{c}
q_{1} \\
q_{1} A \\
\mathrm{M} \\
q_{1} A^{n-1}
\end{array}\right),
$$

where the row vector $\boldsymbol{q}_{1} \in \mathrm{R}^{1 \times n}$ is computed as

$$
\boldsymbol{q}_{1}=\boldsymbol{e}_{n}^{\mathrm{T}} \boldsymbol{R}^{-}
$$

from the controllability matrix $\boldsymbol{R} \in \mathrm{R}^{n \times n}$ of system (1),

$$
\boldsymbol{R}=\left(\begin{array}{lllll}
\boldsymbol{B} & \boldsymbol{A} \boldsymbol{B} & \boldsymbol{A}^{2} \boldsymbol{B} & \Lambda & \boldsymbol{A}^{n-1} \boldsymbol{B}
\end{array}\right),
$$

where $\boldsymbol{e}_{n}=[0, \ldots, 0,1]^{\mathrm{T}} \mathrm{e}_{n}=[0, \ldots, 0,1]^{\mathrm{T}}$ is a unit vector, then the transformed system is transformed into the Frobenius canonical form

$$
\dot{\boldsymbol{z}}=\boldsymbol{A}_{F} \boldsymbol{z}+\boldsymbol{B}_{F} u=\left(\begin{array}{ccccc}
0 & 1 & 0 & \Lambda & 0 \\
0 & 0 & 1 & \Lambda & 0 \\
\mathrm{M} & \mathrm{M} & \mathrm{M} & \mathrm{O} & \mathrm{M} \\
0 & 0 & 0 & \Lambda & 1 \\
-a_{0} & -a_{1} & -a_{2} & \Lambda & -a_{n-1}
\end{array}\right) \boldsymbol{z}+\left(\begin{array}{c}
0 \\
0 \\
0 \\
\mathrm{M} \\
1
\end{array}\right) u \cdot(10)
$$

Then, the system is reduced to a simple and convenient form that can be easily manipulated and we can solve the pole placement problem by state-derivative feedback. If the transformation matrix is nonsingular, then the transformation to the generalized Frobenius canonical form can be made.

\subsection{Solution of the pole placement problem for time-invariant systems}

In this subsection, we will find the state-derivative feedback gain matrix $\boldsymbol{K}$ that assigns the desired closed-loop poles system in a computationally efficient and simple manner. Utilizing the above transformation into Frobenius canonical form, the system can be manipulated by a linear feedback for a desired behavior. By differentiating the transformation equation (5), the resulting closed-loop system in the $z$-coordinate is,

$$
\dot{z}(t)=Q^{-1} \dot{x}(t) .
$$

Hence, after the substitution of (4) and (5) in the above equation we obtain

$$
\dot{z}(t)=Q^{-1}[\boldsymbol{I}+\boldsymbol{B} \boldsymbol{K}]^{-1} \boldsymbol{A} \boldsymbol{Q} z=\boldsymbol{A}_{z} \boldsymbol{z},
$$

where the closed-loop system matrix $\boldsymbol{A}_{z} \in \mathrm{R}^{n \times n}$ is given by

$$
A_{z}=Q^{-1}[I+B K]^{-1} A Q \text {. }
$$

Postmultiply equation (13) by $\boldsymbol{Q}^{-1} \boldsymbol{A}^{-1}[\boldsymbol{I}+\boldsymbol{B} \boldsymbol{K}]$. Hence, we can rewrite the above equation as

$$
A_{z} Q^{-1} A^{-1}[I+B K]=Q^{-1} \text {. }
$$

Given the desired eigenvalues $\left\{\lambda_{1}, \ldots, \lambda_{n}\right\}$. The desired closed-loop characteristic polynomial is,

$$
\begin{aligned}
D(\mathrm{~s}) & =\left(\mathrm{s}-\lambda_{1}\right)\left(\mathrm{s}-\lambda_{2}\right) \ldots\left(\mathrm{s}-\lambda_{n}\right) \\
& =\mathrm{s}^{n}+d_{n-1} \mathrm{~s}^{n-1}+\Lambda+d_{1} \mathrm{~s}+d_{0},
\end{aligned}
$$

where $\boldsymbol{d}=\left[d_{0}, d_{1}, \ldots, d_{n-1}\right]$ are the coefficients of the desired characteristic polynomial. The structure of the desired closed-loop matrix can be written in a canonical form, as

$$
A_{z}=\left(\begin{array}{ccccc}
0 & 1 & 0 & \Lambda & 0 \\
0 & 0 & 1 & \Lambda & 0 \\
\mathrm{M} & \mathrm{M} & \mathrm{M} & \mathrm{O} & \mathrm{M} \\
0 & 0 & 0 & \Lambda & 1 \\
-d_{0} & -d_{1} & -d_{2} & \Lambda & -d_{n-1}
\end{array}\right) .
$$

Equation (14) can be rewritten in terms of the row vectors of the transformation matrix $\boldsymbol{Q}^{-1}$ as,

$$
\boldsymbol{q}_{1} \boldsymbol{A}^{i}[\boldsymbol{I}+\boldsymbol{B} \boldsymbol{K}]=\boldsymbol{q}_{1} \boldsymbol{A}^{i}, \quad i=0, \ldots, n-2,
$$

and 


$$
-\boldsymbol{d} \boldsymbol{Q}^{-1} \boldsymbol{A}^{-1}[\boldsymbol{I}+\boldsymbol{B} \boldsymbol{K}]=\boldsymbol{q}_{1} \boldsymbol{A}^{n-1} .
$$

Based on the definition of the transformation matrix $\boldsymbol{Q}^{-1}$, it is easy to write that

$$
\boldsymbol{q}_{1} \boldsymbol{A}^{i} \boldsymbol{B}=0, \quad i=0, \ldots, n-2 .
$$

Finally, the feedback gain matrix $\boldsymbol{K}$ for the time-invariant system can be written as

$$
\boldsymbol{K}=\left(-\boldsymbol{d} \boldsymbol{Q}^{-1} \boldsymbol{A}^{-1} \boldsymbol{B}\right)^{-1}\left(\boldsymbol{q}_{1} \boldsymbol{A}^{n-1}+\boldsymbol{d} \boldsymbol{Q}^{-1} \boldsymbol{A}^{-1}\right) \text {. }
$$

The above equation can be rewritten as

$$
\boldsymbol{K}=\left(\left(-\sum_{i=0}^{n-1} d_{i} \boldsymbol{q}_{1} \boldsymbol{A}^{i-1}\right) \boldsymbol{B}^{-1}\left(\boldsymbol{q}_{1} \boldsymbol{A}^{n-1}+\sum_{i=0}^{n-1} d_{i} \boldsymbol{q}_{1} \boldsymbol{A}^{i-1}\right)\right. \text {. }
$$

To simplify the feedback gain matrix. First, we utilize the relation

$$
A Q=Q A_{F} .
$$

Then, it is easy to verify that

$$
\boldsymbol{Q}^{-1} \boldsymbol{A}^{-1} \boldsymbol{B}=\boldsymbol{A}_{F}^{-1} \boldsymbol{Q}^{-1} \boldsymbol{B}=\boldsymbol{A}_{F}^{-1} \boldsymbol{B}_{F} .
$$

The inverse of the transformed system matrix $\boldsymbol{A}_{F}$ can be obtained as

$$
\boldsymbol{A}_{F}^{-1}=\left(\begin{array}{ccccc}
-a_{1} / a_{0} & -a_{2} / a_{0} & \Lambda & -a_{n-1} / a_{0} & -1 / a_{0} \\
1 & 0 & \Lambda & 0 & 0 \\
0 & 1 & \mathrm{M} & \mathrm{M} & \mathrm{M} \\
\mathrm{M} & \mathrm{M} & \mathrm{O} & 0 & 0 \\
0 & 0 & \Lambda & 1 & 0
\end{array}\right) .
$$

Finally, it can easily be obtained that

$$
-\boldsymbol{d} \boldsymbol{Q}^{-1} \boldsymbol{A}^{-1} \boldsymbol{B}=-\boldsymbol{d} \boldsymbol{A}_{F}^{-1} \boldsymbol{B}_{F}=-\boldsymbol{d}\left(\begin{array}{c}
-1 / a_{0} \\
0 \\
\mathrm{M} \\
0
\end{array}\right)=\frac{d_{0}}{a_{0}} \text {. }
$$

Then, the feedback gain matrix $\boldsymbol{K}$ for the time-invariant system can be written as

$$
\begin{aligned}
\boldsymbol{K} & =\left(\frac{a_{0}}{d_{0}}\right)\left(\boldsymbol{q}_{1} \boldsymbol{A}^{n-1}+\sum_{i=0}^{n-1} d_{i} \boldsymbol{q}_{1} \boldsymbol{A}^{i-1}\right)= \\
& =\left(\frac{a_{0}}{d_{0}}\right) \boldsymbol{q}_{1} \boldsymbol{A}^{-1}\left[\boldsymbol{A}^{n}+d_{n-1} \boldsymbol{A}^{n-1}+\Lambda+d_{1} \boldsymbol{A}+d_{0} \boldsymbol{I}\right]= \\
& =\left(\frac{a_{0}}{d_{0}}\right) \boldsymbol{e}_{n}^{\mathrm{T}}[\boldsymbol{A} \boldsymbol{R}]^{-1} \boldsymbol{D}(\boldsymbol{A})=\frac{(-1)^{n}}{d_{0}} \boldsymbol{e}_{n}^{\mathrm{T}} \boldsymbol{R}^{-} \operatorname{adj}(\boldsymbol{A}) \boldsymbol{D}(\boldsymbol{A})
\end{aligned}
$$

where $\boldsymbol{D}(\boldsymbol{A})$ denotes the evaluation of the desired characteristic polynomial (15) with the state matrix $A$. The resulting formula for the constant state-derivative feedback gain matrix is the direct analogy of Ackermann formula for traditional state feedback.

The original Ackermann formula has been modified in $[8,9,10]$ into equivalent efficient numerical algorithms for computing the feedback gain matrix $\boldsymbol{K}$. The same can be done for the state-derivative feedback. The resulting equivalent efficient formula based on desired coefficients of the characteristic polynomial is the following recursive procedure

$$
\boldsymbol{K}=\left(\frac{a_{0}}{d_{0}}\right)\left(\boldsymbol{q}_{n}^{\prime}+\sum_{i=0}^{n-1} d_{i} \boldsymbol{q}_{i}^{\prime}\right)
$$

where

$$
\boldsymbol{q}_{0}^{\prime}=\boldsymbol{q}_{1} \boldsymbol{A}^{-1}=\boldsymbol{e}_{n}^{\mathrm{T}}[\boldsymbol{A} \boldsymbol{R}]^{-1}, \boldsymbol{q}_{i}^{\prime}=\boldsymbol{q}_{i-1}^{\prime} \boldsymbol{A}, \quad i=1, \ldots, n .
$$

It is clear that the computation with row vectors is more efficient than with the full square matrices.

Now, if the stabilizing feedback control defined by a set of desired eigenvalues $\lambda_{i}, i=1, \ldots, n$, instead of the coefficients $d_{i}$ of the characteristic equation. Then, the feedback gain matrix is

$$
\begin{aligned}
& \boldsymbol{K}=\frac{a_{0}}{\prod_{i=1}^{n}-\lambda_{i}} q_{1} A^{-} \prod_{i=1}^{n}\left(A-\lambda_{i} \boldsymbol{I}\right)= \\
& =\frac{\operatorname{det}(-\boldsymbol{A})}{\prod_{i=1}^{n} \lambda_{i}} \boldsymbol{e}_{n}^{\mathrm{T}}[\boldsymbol{A R}]^{-1} \prod_{i=1}^{n}\left(\boldsymbol{A}-\lambda_{i} \boldsymbol{I}\right)= \\
& =\frac{1}{\prod_{\lambda_{i=1}^{\lambda}}^{n}} e_{n}^{\mathrm{T}} \boldsymbol{R}^{-1} \operatorname{adj}(\boldsymbol{A}) \prod_{i=1}^{n}\left(\boldsymbol{A}-\lambda_{i} \boldsymbol{I}\right)
\end{aligned}
$$

And again utilizing the above simplification (26), the above equation can be written as

$$
\boldsymbol{K}=\frac{a_{0}}{\prod_{i=1}^{n} \lambda_{i}} \boldsymbol{q}_{n}^{\prime},
$$

where

$$
\boldsymbol{q}_{0}^{\prime}=\boldsymbol{q}_{1} \boldsymbol{A}^{-1}=\boldsymbol{e}_{n}^{\mathrm{T}}[\boldsymbol{A R}]^{-1}, \boldsymbol{q}_{i}^{\prime}=\boldsymbol{q}_{i-1}^{\prime}\left(\boldsymbol{A}-\lambda_{i} \boldsymbol{I}\right), i=1, \ldots, n .
$$

One can easily note that the proposed algorithm is simple and easy to implement. In addition, the state-derivative feedback gain matrix calculations are not done in the intermidiate domain and direct implementation is performed in the original state space. We do not need to compute the transformation into the generalized Frobenius form. One of the main advantages of the transformation matrix is the posibility to easily derive an explicit analytic expression for the feedback gain matrix. The above algorithm is valid for desired eigenvalues that are either real or complex-conjugate poles.

From the derivation of the state-derivative feedback pole placement the necessary and sufficient conditions for arbitrary pole placement can be described. For the transformation into the Frobenius canonical form and/or computing formulas (25)-(28) the controllability matrix $\boldsymbol{R}$ must be of full rank, i.e. the original system must be controllable as for traditional state feedback. In addition, the coefficient $a_{0}$ must be non-zero. Using our knowledge about the coefficients of the characteristic polynomial the coefficients is equivalent to the condition that

$$
a_{0}=\prod_{i=1}^{n}-\lambda_{O i} \neq 0
$$

where here $\lambda_{O i},(i=1, \ldots, n)$, are the original poles of the system (1). This means that all the original poles must be non-zero. This is equivalent according to $[20,1.1 .7]$ to the 
condition that the system matrix $\boldsymbol{A}$ of the original system (1) is nonsingular (it has full rank $n$ ).

Based on the above necessary and sufficient conditions, we are now in the position to present the first main result of the present work.

\section{Theorem 1:}

Consider the controllable single-input time-invariant linear system of (1). If matrix $\boldsymbol{A}$ is non-singular, then the poles of system (1) can be arbitrarily placed in the desired places by the state-derivative feedback (3) using the constant feedback gain matrix $\boldsymbol{K}$ computed by one of the formulas (25)-(28).

It is clear that this solution is general, it requires no iteration and can be easily directly computed. This solves the pole placement for a linear time-invariant system by state-derivative feedback. Another interesting feature is that in many cases the state-derivative feedback gains (26)-(28) are in absolute values smaller than the traditional state feedback gains $[8,9,10]$ if the inverse of system matrix $\boldsymbol{A}$ reduces the gains in (26)-(28).

The state-derivative feedback is a derivative feedback. It can modify the system dynamics, but it cannot modify the steady-state errors. The steady-state error is finite for the original system, as system matrix $\boldsymbol{A}$ is nonsingular and it remains unchanged by the state-derivative feedback.

\section{Pole placement by state-derivative feedback for linear time-varying systems}

The above methodology can be extended for general single-input controllable linear time-varying systems

$$
\dot{x}(t)=\boldsymbol{A}(t) \boldsymbol{x}(t)+\boldsymbol{B}(t) u(t), \boldsymbol{x}\left(t_{0}\right)=x_{0}
$$

where $x(t) \in \mathrm{R}^{n}$ and $u(t) \in \mathrm{R}$ are the state vector and the scalar control input, respectively, while $\boldsymbol{A}(t) \in \mathrm{R}^{n \times n}$ and $\boldsymbol{B}(t) \in \mathrm{R}^{n}$ are the system matrix and control gain vector, respectively. The sufficient condition for the existence and unique solution is to require that all elements of $\boldsymbol{A}(t)$ be continuous in the time interval of interest $t \in\left[t_{0}, \infty\right]$. The objective here is to find a time-dependent linear feedback gain matrix that will stabilize the system. Then, this system can be stabilized by the varying state-derivative feedback control law,

$$
u(t)=-\boldsymbol{K}(t) \dot{x}(t),
$$

and the closed-loop system can be written as

$$
\dot{\boldsymbol{x}}(t)=[\boldsymbol{I}+\boldsymbol{B}(t) \boldsymbol{K}(t)]^{-1} \boldsymbol{A}(t) \boldsymbol{x} .
$$

The objective now is to construct the varying feedback gain matrix $\boldsymbol{K}(t)$ in order to stabilize the system. In this treatment, we turn our attention to utilize the Frobenius transformation as an intermediate step to simplify the pole placement problem. Let us take the following time-varying state transformation

$$
z=Q^{-1}(t) x, x=Q(t) z
$$

Then, the system is transformed to the Frobenius canonical form and the dynamic system matrices can be computed as

$$
A_{F}(t)=Q^{-1}(A Q-\dot{Q}), B_{F}=Q^{-1} B
$$

where $\boldsymbol{A}_{F}(t) \in \mathrm{R}^{n \times n}$ and $\boldsymbol{B}_{F} \in \mathrm{R}^{n}$ are the transformed system matrix and control gain vector, respectively. The transformed system is the same as (10), however with $\boldsymbol{a}(t)=\left[a_{0}(t), a_{1}(t), \ldots, a_{n-1}(t)\right]$ being the time-varying coefficients. Note that the eigenvalues of the time varying dynamic system do not have any meaning regarding its behaviour or its stability features.

The state transformation matrix $\boldsymbol{Q}^{-1}(t) \in \mathrm{R}^{n \times n}$ can be calculated as follows

$$
Q^{-1}(t)=\operatorname{rows}\left(\begin{array}{llll}
\boldsymbol{q}_{1} & \boldsymbol{q}_{2} & \Lambda & \boldsymbol{q}_{n}
\end{array}\right)
$$

where $\boldsymbol{q}_{i} \in \mathrm{R}^{1 \times n}$ is computed by using the recursive computations of the rows as follows

$$
\boldsymbol{q}_{1}=\boldsymbol{e}_{n}^{\mathrm{T}} \boldsymbol{R}^{-1}, \boldsymbol{q}_{i+1}=\boldsymbol{q}_{i} \boldsymbol{A}+\dot{\boldsymbol{q}}_{i}, i=1, \ldots, n-1 .
$$

The controllability matrix for the time-varying system $\boldsymbol{R}(t) \in \mathrm{R}^{n \times n}$ is formed as

$$
\boldsymbol{R}(t)=\left(\begin{array}{llll}
\boldsymbol{r}_{1} & \boldsymbol{r}_{2} & \Lambda & \boldsymbol{r}_{n}
\end{array}\right)
$$

where $\boldsymbol{r}_{i} \in \mathrm{R}^{n}$ can be computed algebraically using the recursion

$$
\boldsymbol{r}_{1}=\boldsymbol{B}, \boldsymbol{r}_{i+1}=\boldsymbol{A} \boldsymbol{r}_{i}-\dot{\boldsymbol{r}}_{i}, i=1, \ldots, n-1 .
$$

If $\boldsymbol{Q}(t), \boldsymbol{Q}^{-1}(t)$, and $\dot{\boldsymbol{Q}}(t)$ are continuous and bounded matrices and $Q^{-1}(t)$ has a full rank at the time interval of interest, $t \in\left[t_{0}, \infty\right]$, then this transformation is called a Lyapunov transformation. Note that the Lyapunov transformation means that the transformation from one system to the other preserves the property of stability. Consequently, this ensures that we can stabilize the time-varying system by means of placing the poles of the Lyapunov equivalent by a linear time-invariant system.

Assuming that the above transformation to the Frobenius canonical form is of the Lyapunov kind then the pole placement technique from the time-invariant case can be extended for the time-varying case. By differeintiating the transformation equation (33) and substitute (32), the resulting closed-loop system in the $z$-coordinate is,

$$
\begin{aligned}
\dot{z} & =\boldsymbol{Q}^{-1} \dot{\boldsymbol{x}}+\dot{\boldsymbol{Q}}^{-1} \boldsymbol{x}= \\
& =\left(\dot{\boldsymbol{Q}}^{-1}+\boldsymbol{Q}^{-1}[\boldsymbol{I}+\boldsymbol{B} \boldsymbol{K}]^{-1} \boldsymbol{A}\right) \boldsymbol{Q} z=A_{z} z,
\end{aligned}
$$

where $\boldsymbol{A}_{z} \in \mathrm{R}^{n \times n}$ is the closed-loop system matrix and is given as (16), and can be computed as

$$
\boldsymbol{A}_{z}=\left(\dot{\boldsymbol{Q}}^{-1}+\boldsymbol{Q}^{-1}[\boldsymbol{I}+\boldsymbol{B} \boldsymbol{K}]^{-1} \boldsymbol{A}\right) \boldsymbol{Q} \text {. }
$$

Hence, we can rewrite the above equation as

$$
\left(\boldsymbol{A}_{z} \boldsymbol{Q}^{-1}-\dot{\boldsymbol{Q}}^{-1}\right) \boldsymbol{A}^{-1}[\boldsymbol{I}+\boldsymbol{B} \boldsymbol{K}]=\boldsymbol{Q}^{-1} \text {. }
$$

Applying the same procedure for the time-invariant system, it is easy to write the $n$ equations describing the system in terms of the row vectors $\boldsymbol{q}_{i}, i=1, \ldots, n$ of $\boldsymbol{Q}^{-1}(t)$ as,

$$
\left(\boldsymbol{q}_{i+1}-\dot{\boldsymbol{q}}_{i}\right) \boldsymbol{A}^{-1}[\boldsymbol{I}+\boldsymbol{B} \boldsymbol{K}]=\boldsymbol{q}_{i}, i=1, \ldots, n-1
$$

and

$$
\left(-\boldsymbol{d} \boldsymbol{Q}^{-1}-\dot{\boldsymbol{q}}_{n}\right) \boldsymbol{A}^{-1}[\boldsymbol{I}+\boldsymbol{B K}]=\boldsymbol{q}_{n},
$$

Then, the feedback gain matrix $\boldsymbol{K}(t)$ for the time-varying system can be written as 
$\boldsymbol{K}(t)=\left[\left(-d Q^{-1}-\dot{\boldsymbol{q}}_{n}\right) \boldsymbol{A}^{-1} \boldsymbol{B}\right]^{-1}\left[\boldsymbol{q}_{n}+\left(\boldsymbol{d} Q^{-1}+\dot{\boldsymbol{q}}_{n}\right) \boldsymbol{A}^{-1}\right]$.

The feedback gain matrix $\boldsymbol{K}(t)$ can be rewritten as

$$
\begin{gathered}
\boldsymbol{K}(t)=\left(1-\left(\boldsymbol{q}_{n+1}+\sum_{i=0}^{n-1} d_{i} \boldsymbol{q}_{i+1}\right) \boldsymbol{A}^{-1} \boldsymbol{B}\right)^{-} . \\
\left(\boldsymbol{q}_{n+1}+\sum_{i=0}^{n-1} d_{i} \boldsymbol{q}_{i+1}\right) \boldsymbol{A}^{-1} .
\end{gathered}
$$

Next, we consider a stabilizing feedback control defined by the desired eigenvalues $\left\{\lambda_{1}, \ldots, \lambda_{n}\right\}$. The gain matrix is directly computed by an efficient numerical algorithm as

$\boldsymbol{K}(t)=\left(1-\boldsymbol{q}_{n+1}^{\prime} \boldsymbol{A}^{-1} \boldsymbol{B}\right)^{-1} \boldsymbol{q}_{n+1}^{\prime} \boldsymbol{A}^{-1}$,

where

$\boldsymbol{q}_{1}^{\prime}=\boldsymbol{q}_{1}=\boldsymbol{e}_{n}^{\mathrm{T}} \boldsymbol{R}^{-1}, \boldsymbol{q}_{i+1}^{\prime}=\boldsymbol{q}_{i}^{\prime}\left(\boldsymbol{A}-\lambda_{i} \boldsymbol{I}\right)+\dot{\boldsymbol{q}}_{i}, i=1, \ldots, n$.

From the derivation of the state-derivative feedback pole plaement the necessary and sufficient conditions for arbitrary pole placement can be described. For the transformation into the Frobenius canonical form and/or computing formulas (34)-(38) the controllability matrix $\boldsymbol{R}(t)$ and the transformation matrix $\boldsymbol{Q}^{-1}(t)$ must be of full rank, the matrices $\boldsymbol{Q}(t)$, $\boldsymbol{Q}^{-1}(t), \dot{\boldsymbol{Q}}(t)$ are continuous and bounded in order for the transformation be of the Lyapunov kind. In addition, matrix $\boldsymbol{A}(t)$ is continuous, nonsingular, and its inverse is bounded. Finally, the term . Everything must be valid at the time interval of interest, $t \in\left[t_{0}, \infty\right]$.

Now, the following theorem can be presented for the single-input time-varying control systems.

\section{Theorem 2:}

Consider the controllable single-input time-varying linear system of (30). If matrix $\boldsymbol{A}(t)$ is continuous, nonsingular and its inverse is bounded. Furthermore, the transformation matrix $Q^{-1}(t)$ is a transformation of a Lyapunov kind and the term $\boldsymbol{q}_{n+1}^{\prime} \boldsymbol{A}^{-1} \boldsymbol{B} \neq 1$ for the time interval of interest $t \in\left[t_{0}, \infty\right]$. Then the poles of the system (30) can be arbitrarily placed in the desired places by the state-derivative feedback (31), using the time-varying feedback gain matrix $\boldsymbol{K}(t)$ computed by one of the formulas (44)-(45).

With the above development, it is clear that this solution is general, it requires no iteration and can be relatively easily directly computed. We do not need to complete the transformation into a generalized Frobenius form. Furthermore, the characteristic polynomial coefficients or eigenvalues do not have to be calculated. This solves the pole placement for a linear time-varying system by state-derivative feedback. The procedure defined here represents a unique treatment for state-derivative feedback in the literature.

\section{Illustrative examples}

In this section, simulation results are given to demonstrate the feasibility and effectiveness of the proposed pole placement algorithm by state-derivative feedback.

\section{Example 1}

The configuration of the mechanical system and its parameters are shown in Fig. 1. The dynamic equation of this system can be described in the state-space form as

$$
\dot{x}=\left(\begin{array}{cccc}
0 & 0 & 1 & 0 \\
0 & 0 & 0 & 1 \\
\frac{-k_{1}-k_{2}}{m_{1}} & \frac{k_{2}}{m_{1}} & \frac{-b_{1}-b_{2}}{m_{1}} & \frac{b_{2}}{m_{1}} \\
\frac{k_{2}}{m_{2}} & \frac{-k_{2}}{m_{2}} & \frac{b_{2}}{m_{2}} & \frac{-b_{2}}{m_{2}}
\end{array}\right) \boldsymbol{x}+\left(\begin{array}{c}
0 \\
0 \\
\frac{-1}{m_{1}} \\
\frac{1}{m_{2}}
\end{array}\right)
$$

where $m_{1}$ and $m_{2}$ are the first and second mass, $k_{1}$ and $k_{2}$ are the spring constants, $b_{1}$ and $b_{2}$ are the damper constants, $x_{1}$ and $x_{2}$ are the first and second mass vertical displacement, the state vector $x=\left[\begin{array}{llll}x_{1} & x_{2} & \dot{x}_{1} & \dot{x}_{2}\end{array}\right]$, and $u$ is the control input.

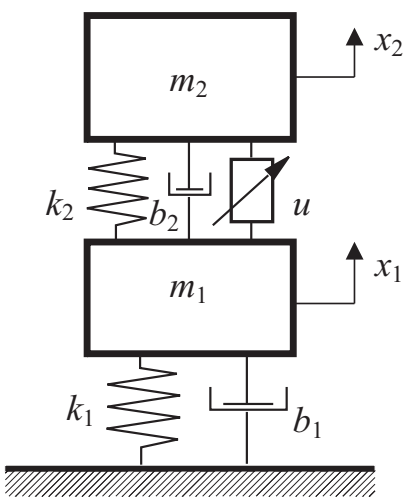

Fig. 1: Mechanical system

The model parameters are taken as $m_{1}=100 \mathrm{~kg}$; $m_{2}=10 \mathrm{~kg} ; k_{1}=360 \mathrm{kN} / \mathrm{m} ; k_{2}=36 \mathrm{kN} / \mathrm{m} ; b_{1}=70 \mathrm{~N} \cdot \mathrm{s} / \mathrm{m}$, and $b_{2}=50 \mathrm{~N} \cdot \mathrm{s} / \mathrm{m}$. The transformation matrix

$\boldsymbol{Q}^{-1}=\left(\begin{array}{cccc}0.02778 & 0.00278 & 0 & 0 \\ 0.19444 & 0 & 0.02778 & 0.00278 \\ -100 & 0 & 0 & 0 \\ 0 & 0 & -100 & 0\end{array}\right)$

while the equivalent Frobenius canonical form has a state description as

$$
\dot{\boldsymbol{x}}=\left(\begin{array}{cccc}
0 & 1 & 0 & 0 \\
0 & 0 & 1 & 0 \\
0 & 0 & 0 & 1 \\
-1.296 \cdot 10^{7} & -20520 & -7563.5 & -6.2
\end{array}\right) z+\left(\begin{array}{l}
0 \\
0 \\
0 \\
1
\end{array}\right) u
$$

with the coefficients of the characteristic polynomial $\boldsymbol{a}=\left[1.296 \cdot 10^{7}, 20520,7563.5,6.2\right]$. For this system the open-loop poles are $-2.1835 \pm 70.1294 \mathrm{i}$ and $-0.9165 \pm 51.3006 \mathrm{i}$. The desired closed-loop poles are selected as $-5 \pm 2 \mathrm{i}$ and $-10 \pm 5 \mathrm{i}$.

Let us apply the control synthesis procedure of pole placement from the previous sections to this system. The computed state-derivative feedback gain matrix is

$\boldsymbol{K}=10^{5} \cdot[-105.19,0.18117,-3.2225,0.0349]$. 
To verify the design a simulation was performed. The initial conditions of the states are taken as $x\left(t_{0}\right)=[0.5,0.5,0.2,0.2]^{\mathrm{T}}$. The transient responses of states and control input are displayed in Fig. 2.
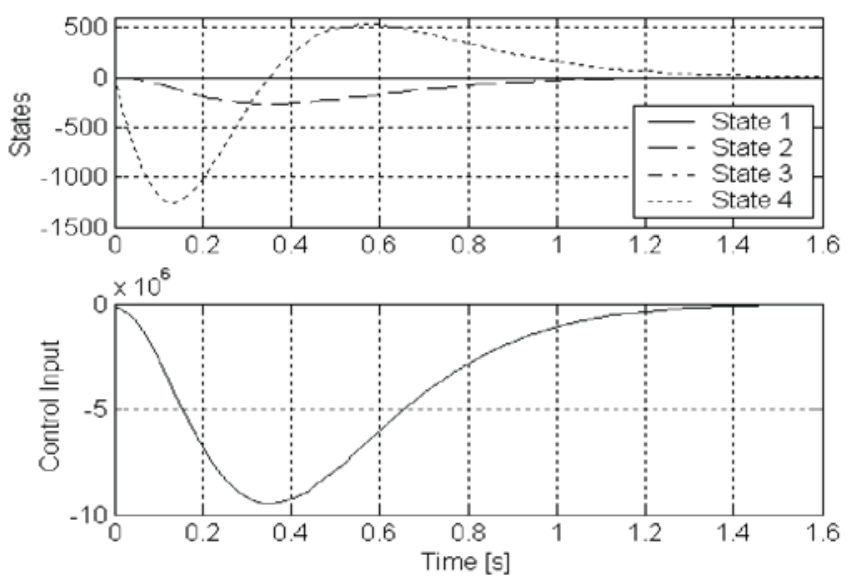

Fig. 2: Transient response and control input of the system controlled by state-derivative feedback

For a comparison, the Ackermann gain matrix for the same desired system poles is

$\boldsymbol{K}_{\mathrm{S}}=10^{5} \cdot[3.6074,-0.3599,-0.02829,-0.00045]$.

The stabilized results for the same initial conditions are plotted in Fig. 3. They are the same as with state-derivative feedback in both transient response and control input.
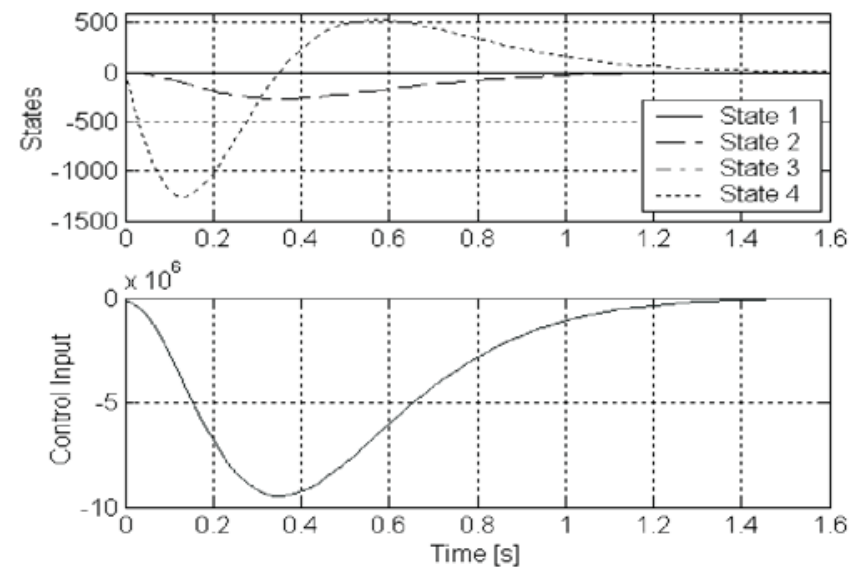

Fig. 3: Transient response and control input of the system controlled by the Ackermann formula

\section{Example 2}

Consider the familiar ball and beam system. Fig. 4 presents the configuration of this system and its parameters. The dynamic equations of the linearized state space model for small motions about $\theta=0.0$, and $r=r_{0}$ is

$\dot{\boldsymbol{x}}=\left(\begin{array}{cccc}0 & 1 & 0 & 0 \\ 0 & \frac{-b}{m r_{0}^{2}+I} & \frac{-m g}{m r_{0}^{2}+I} & 0 \\ 0 & 0 & 0 & 1 \\ \frac{-m g \rho^{2}}{m \rho^{2}+I_{b}} & 0 & 0 & 0\end{array}\right) \boldsymbol{x}+\left(\begin{array}{c}0 \\ \frac{1}{m r_{0}^{2}+I} \\ 0 \\ 0\end{array}\right) u$

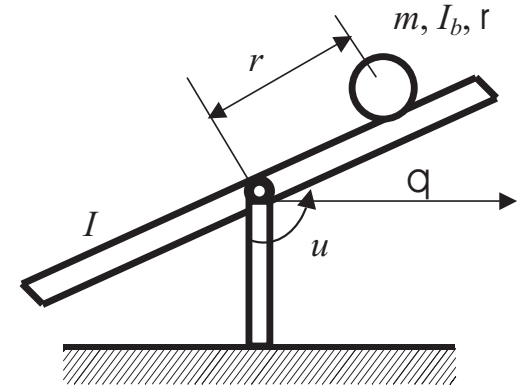

Fig. 4: Ball and beam system

where $m, I_{b}$, and $\rho$ are the mass, moment of inertia and radius of the ball, respectively, $I$ is the beam moment of inertia, $g$ is the gravitational acceleration, $b$ is the coefficient of viscous friction opposing the beam rotation, $\theta$ is the angle that the beam makes with the horizontal, $r$ is the distance between the center of the ball and center of rotation of the beam, the state vector $\boldsymbol{x}=\left[\begin{array}{llll}\theta & \dot{\theta} & r & \dot{r}\end{array}\right]$, and $u$ is the control torque input. In this treatment, we assume that the ball is frictionless rolling along the beam. The control objective is to balance the ball at a distance from the central pivot point by tilting the beam back and forth using the motor. The values of various parameters of the model are $m=1 \mathrm{~kg} ; \rho=0.05 \mathrm{~m} ; r_{0}=0.1 \mathrm{~m}$; $g=9.81 \mathrm{~m} / \mathrm{s}^{2} ; b=0.1 \mathrm{~N} \cdot \mathrm{s} / \mathrm{m}, I_{b}=5 \mathrm{~kg} \cdot \mathrm{m}^{2}$, and $I=1 \mathrm{~kg} \cdot \mathrm{m}^{2}$. The characteristic polynomial coefficients are $\boldsymbol{a}=[-0.0479$, $0.0,0.0,0.020]$. The original poles are, $+0.4629,-0.4729$ and $-0.005 \pm 0.4678$ i. Obviously, the open loop system is unstable. The desired closed-loop poles are $-7 \pm 2 \mathrm{i}$ and $-10 \pm 5 \mathrm{i}$. The state-derivative feedback gain matrix is computed as,

$$
\boldsymbol{K}=[-0.1012,-5.01,4.1609,0.6782] .
$$

Taking the initial states as $x\left(t_{0}\right)=[0.1,0.1,0.05,0.05]^{\mathrm{T}}$. The simulation results are shown in Fig. 5 .

Compared with the Ackermann formula, the gain matrix for the same desired system poles is

$$
\boldsymbol{K}_{\mathrm{s}}=10^{5} \cdot[0.023,0.002,-13.568,-5.755] .
$$

Similar to the results of the previous example, the performance of the two cases are the same in transient response and control input.
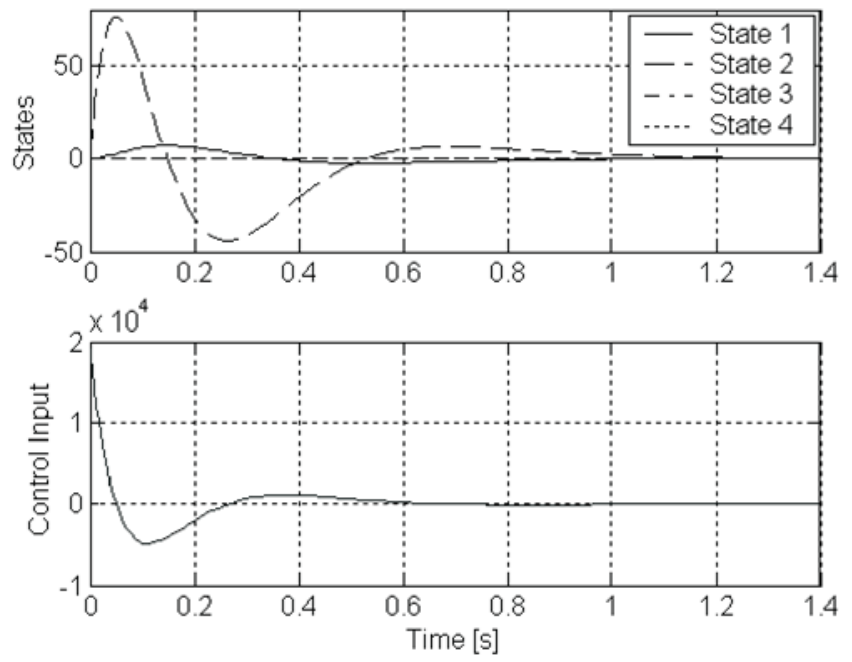

Fig. 5: Transient response and control input of the system controlled by state-derivative feedback 


\section{Example 3}

Consider the dynamic equation of the single-input time-varying system

$$
\dot{x}(t)=\left(\begin{array}{ccc}
0.1 e^{-t} & 0.2 & 0 \\
0.1 & 0.2 & 0.1 e^{-t} \\
-0.1 & 0 & 0.1
\end{array}\right) x(t)+\left(\begin{array}{c}
0 \\
0.1 \\
0
\end{array}\right) u(t) .
$$

This system is unstable and the zero-input transient response of an open-loop system are shown in Fig. 6.

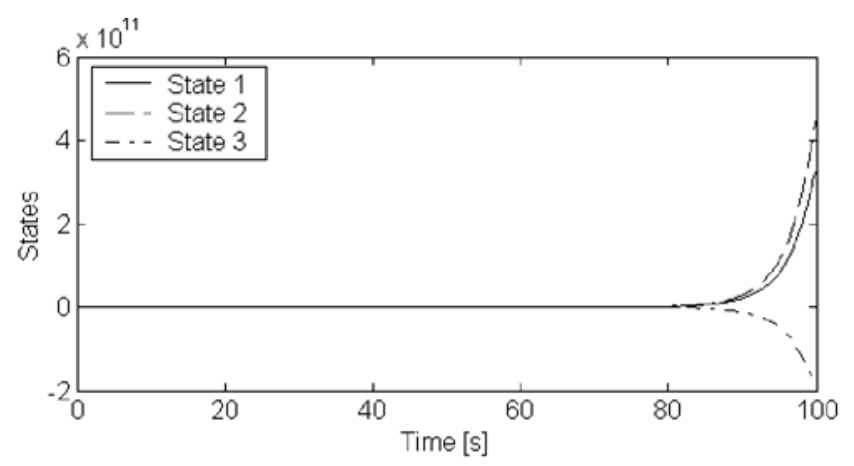

Fig. 6: Zero-input transient response of an open-loop system

To stabilize the above system, the pole placement technique is used. First, the controllability matrix and its inverse are computed as

$$
\boldsymbol{R}(t)=\left(\begin{array}{ccc}
0 & 0.02 & 0.002\left(\mathrm{e}^{-t}+2\right) \\
0.1 & 0.02 & 0.006 \\
0 & 0 & -0.002
\end{array}\right)
$$

and

$$
\boldsymbol{R}^{-1}(t)=\left(\begin{array}{ccc}
-10 & 10 & -10\left(\mathrm{e}^{-t}-1\right) \\
50 & 0 & 50\left(\mathrm{e}^{-t}+2\right) \\
0 & 0 & -500
\end{array}\right)
$$

It is clear that the controllability matrix is a full rank in the time interval of interest $t \in\left[t_{0}, \infty\right]$. This means the system is completely state controllable.

Now, the transformation matrix can be computed as follows. The rows $\boldsymbol{q}_{i}, i=1, \ldots, n$ are computed as

$$
\boldsymbol{q}_{1}=\boldsymbol{e}_{n}^{\mathrm{T}} \boldsymbol{R}^{-1}=\left(\begin{array}{lll}
0 & 0 & -500
\end{array}\right)
$$

$$
\boldsymbol{q}_{2}=\boldsymbol{q}_{1} \boldsymbol{A}+\dot{\boldsymbol{q}}_{1}=\left(\begin{array}{lll}
50 & 0 & -50
\end{array}\right)
$$

and

$$
\boldsymbol{q}_{3}=\boldsymbol{q}_{2} \boldsymbol{A}+\dot{\boldsymbol{q}}_{2}=\left(\begin{array}{lll}
5\left(\mathrm{e}^{-t}+1\right) & 10 & -5
\end{array}\right) .
$$

Then, the transformation matrix, inverse, and derivative are

$$
\begin{aligned}
& \boldsymbol{Q}^{-1}(t)=\left(\begin{array}{ccc}
0 & 0 & -500 \\
50 & 0 & -50 \\
5\left(\mathrm{e}^{-t}+1\right) & 10 & -5
\end{array}\right), \\
& \boldsymbol{Q}(t)=\left(\begin{array}{ccc}
-0.002 & 0.02 & 0 \\
0.001 \mathrm{e}^{-t} & -0.01\left(\mathrm{e}^{-t}+1\right) & 0.1 \\
-0.002 & 0 & 0
\end{array}\right), \\
& \dot{\boldsymbol{Q}}^{-1}(t)=\left(\begin{array}{ccc}
0 & 0 & 0 \\
0 & 0 & 0 \\
-5 \mathrm{e}^{-t} & 0 & 0
\end{array}\right)
\end{aligned}
$$

and

$$
\dot{\boldsymbol{Q}}(t)=\left(\begin{array}{ccc}
0 & 0 & 0 \\
-0.001 \mathrm{e}^{-t} & -0.001 \mathrm{e}^{-t} & 0 \\
0 & 0 & 0
\end{array}\right) .
$$

These matrices are continuous and bounded and the transformation matrix has a full rank at the time interval of interest $t \in\left[t_{0}, \infty\right]$. Then this transformation is a Lyapunov transformation and the proposed pole placement technique can be applied.

The transformed system matrix $\boldsymbol{A}_{F}(t)$ and the control gain vector $\boldsymbol{B}_{F}$ are

$$
A_{F}(t)=Q^{-1}(A Q-\dot{Q})
$$

$$
=\left(\begin{array}{ccc}
0 & 1 & 0 \\
0 & 0 & 1 \\
0.01\left(\mathrm{e}^{-t}-0.2\right) & -0.13 \mathrm{e}^{-t} & 0.1\left(\mathrm{e}^{-t}+3\right)
\end{array}\right) \text {, }
$$

and

$$
\boldsymbol{B}_{F}=\boldsymbol{Q}^{-1} \boldsymbol{B}=\left(\begin{array}{l}
0 \\
0 \\
1
\end{array}\right),
$$

with the time-varying coefficients

$$
\boldsymbol{a}(t)=\left(-0.01\left(\mathrm{e}^{-t}-0.2\right) \quad 0.13 \mathrm{e}^{-t}-0.1\left(\mathrm{e}^{-t}+3\right)\right)
$$

The computation of the feedback gain matrix for the desired eigenvalues $\lambda_{1}, \lambda_{2}, \lambda_{3}$ can be done as follows. First, the rows $\boldsymbol{q}_{1}^{\prime}$, $i=1, \ldots, n+1$ are

$$
\begin{aligned}
& \boldsymbol{q}_{1}^{\prime}=\boldsymbol{q}_{1}=\left(\begin{array}{lll}
0 & 0 & -500
\end{array}\right), \\
& \boldsymbol{q}_{2}^{\prime}=\boldsymbol{q}_{1}^{\prime}\left(\boldsymbol{A}-\lambda_{1} \boldsymbol{I}\right)+\dot{\boldsymbol{q}}_{1}=\left(\begin{array}{lll}
50 & 0 & -500\left(0.1-\lambda_{1}\right)
\end{array}\right) \text {, } \\
& \boldsymbol{q}_{3}^{\prime}=\boldsymbol{q}_{2}^{\prime}\left(\boldsymbol{A}-\lambda_{2} \boldsymbol{I}\right)+\dot{\boldsymbol{q}}_{2}=\left(50\left(0.1 \mathrm{e}^{-t}-\lambda_{1}-\lambda_{2}+0.1\right) \quad 10 \quad-500\left(0.1-\lambda_{1}\right)\left(0.1-\lambda_{1}\right)\right) \text {, }
\end{aligned}
$$

and

$$
\begin{aligned}
\boldsymbol{q}_{4}^{\prime}=\boldsymbol{q}_{3}^{\prime}\left(\boldsymbol{A}-\lambda_{3} \boldsymbol{I}\right)+\dot{\boldsymbol{q}}_{3}= & \left(0.5 \mathrm{e}^{-2 t}-4.5 \mathrm{e}^{-t}-5\left(\lambda_{1}+\lambda_{2}+\lambda_{3}\right)\left(\mathrm{e}^{-t}+1\right)+50\left(\lambda_{1} \lambda_{2}+\lambda_{1} \lambda_{3}+\lambda_{2} \lambda_{3}\right)+1.5,\right. \\
& \left.\mathrm{e}^{-t}-10\left(\lambda_{1}+\lambda_{2}+\lambda_{3}\right)+3, \quad \mathrm{e}^{-t}-500\left(0.1-\lambda_{1}\right)\left(0.1-\lambda_{2}\right)\left(0.1-\lambda_{3}\right)\right)
\end{aligned}
$$


Then, we can compute

$$
\begin{aligned}
\boldsymbol{q}_{4}^{\prime} A^{-1}= & \left(55 \mathrm{e}^{-t}-5000 \lambda_{1} \lambda_{2} \lambda_{3}-50\left(\lambda_{1}+\lambda_{2}+\lambda_{3}\right)+5, \quad-50 \mathrm{e}^{-t}+5000 \lambda_{1} \lambda_{2} \lambda_{3}+10,\right. \\
& \left.50 \mathrm{e}^{-2 t}-5000 \lambda_{1} \lambda_{2} \lambda_{3}\left(\mathrm{e}^{-t}+1\right)+50\left(\lambda_{1}+\lambda_{2}+\lambda_{3}\right)-500\left(\lambda_{1} \lambda_{2}+\lambda_{1} \lambda_{3}+\lambda_{2} \lambda_{3}\right)-5\right),
\end{aligned}
$$

and

$$
\boldsymbol{q}_{4}^{\prime} \boldsymbol{A}^{-1} \boldsymbol{B}=5 \mathrm{e}^{-t}+500 \lambda_{1} \lambda_{2} \lambda_{3}+1 .
$$

Finally, the feedback gain matrix is

$$
\begin{aligned}
& \boldsymbol{K}(t)=\left(1-\boldsymbol{q}_{4}^{\prime} \boldsymbol{A}^{-1} \boldsymbol{B}\right) \boldsymbol{q}_{4}^{\prime} \boldsymbol{A}^{-1}=\left(10+\frac{\mathrm{e}^{-t}-10\left(\lambda_{1}+\lambda_{2}+\lambda_{3}\right)+1}{\mathrm{e}^{-t}-100 \lambda_{1} \lambda_{2} \lambda_{3}}, \quad-10 \frac{2}{\mathrm{e}^{-t}-100 \lambda_{1} \lambda_{2} \lambda_{3}},\right. \\
& \left.10 \mathrm{e}^{-t}+\frac{1000 \lambda_{1} \lambda_{2} \lambda_{3}-100\left(\lambda_{1} \lambda_{2}+\lambda_{1} \lambda_{3}+\lambda_{2} \lambda_{3}\right)+10\left(\lambda_{1}+\lambda_{2}+\lambda_{3}\right)+1}{\mathrm{e}^{-t}-100 \lambda_{1} \lambda_{2} \lambda_{3}}\right) .
\end{aligned}
$$

Our selection of the desired closed-loop poles are $-5 \pm 2 \mathrm{i}$ and -7 . Applying the pole placement technique to this system, the initial condition of the states is selected as $x\left(t_{0}\right)=[-1,1,2]^{\mathrm{T}}$. The transient response and control input are shown in Fig. 7. In addition, the elements of the gain matrix are displayed in Fig. 8.

As a comparison with the state feedback, the state feedback gain matrix for this system can be computed as [8, 9],

$$
\begin{aligned}
\boldsymbol{K}_{\mathrm{S}}(t)=\boldsymbol{q}_{4}^{\prime}= & \left(0.5 \mathrm{e}^{-2 t}-4.5 \mathrm{e}^{-t}-5\left(\lambda_{1}+\lambda_{2}+\lambda_{3}\right)\left(\mathrm{e}^{-t}+1\right)+50\left(\lambda_{1} \lambda_{2}+\lambda_{1} \lambda_{3}+\lambda_{2} \lambda_{3}\right)+1.5,\right. \\
& \left.\mathrm{e}^{-t}-10\left(\lambda_{1}+\lambda_{2}+\lambda_{3}\right)+3, \quad \mathrm{e}^{-t}-500\left(0.1-\lambda_{1}\right)\left(0.1-\lambda_{2}\right)\left(0.1-\lambda_{3}\right)\right) .
\end{aligned}
$$

Taking the desired eiginvalues and the same initial conditions for the state-derivative feedback, the simulation results indicate the same transient performance and control input with lower gains. The elements of the gain matrix are displayed in Fig. 9.
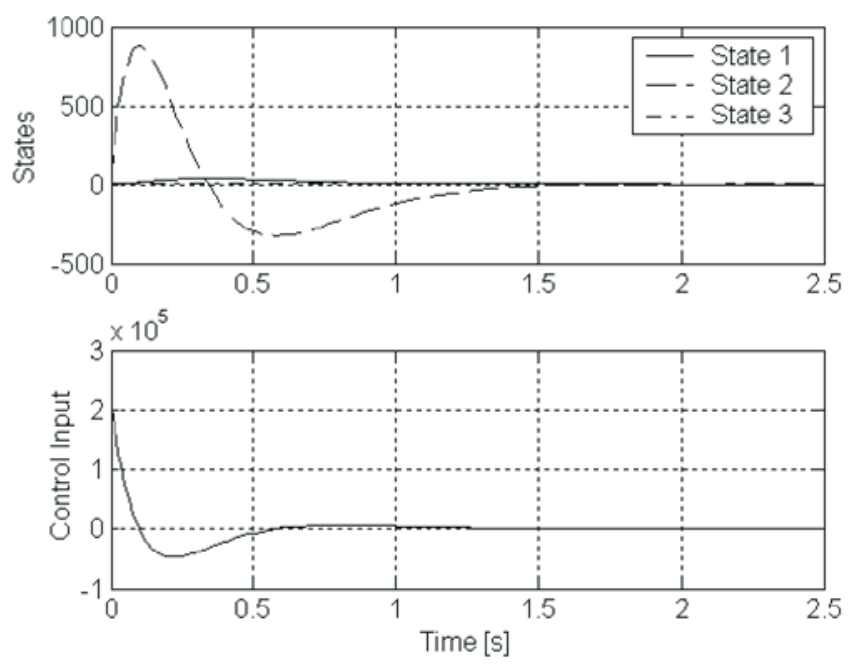

Fig. 7: Transient response and control input of a time-varying system controlled by state-derivative feedback

Based on the simulation analysis above, we note the great reduction in the state-derivative feedback gain matrix compared to the well-known state feedback approach, with the same performance for time-invariant and time-varying systems.

This paper shows how the pole placement approach can be used to design a controller-based state-derivative feedback control which yields a closed-loop system with specified char-

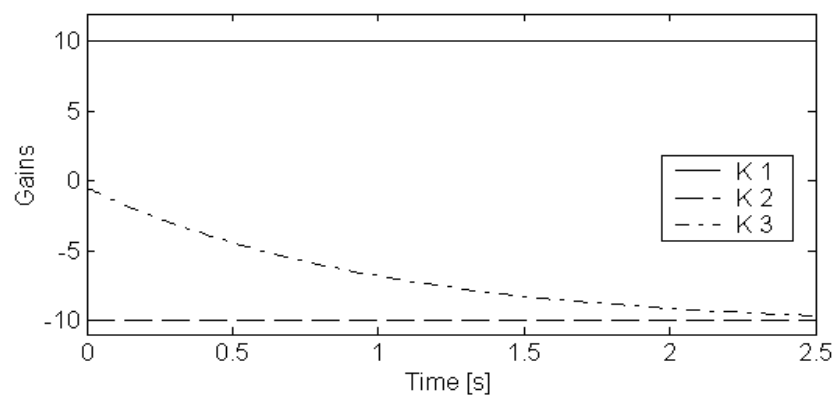

Fig. 8: Gain elements of a time-varying system controlled by state-derivative feedback

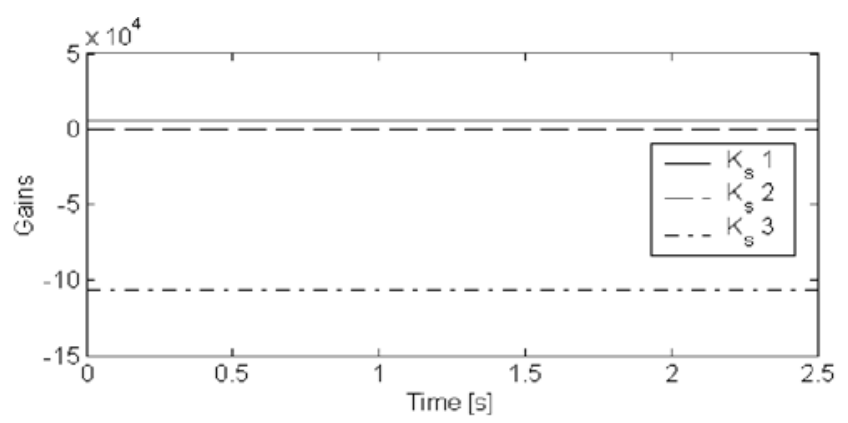

Fig. 9: Gain elements of a time-varying system controlled by state feedback

acteristics. The approach is relevant for design with perservation of stability when some necessary and sufficient conditions are provided. Compared with a well-known state feedback, the state-derivative feedback controller in some cases achieves the same performance with a lower gain. 


\section{Conclusions}

This paper has generally formulated and proposed a new concept and technique for solving the pole placement problem by full state-derivative feedback for a linear time-invariant and time-varying single-input system. There have been formulated necessary and sufficient conditions for solving such pole placement. The resulting formula for the time-invariant case is a generalization of the Ackermann formula for traditional state feedback. The described algorithm avoids previous iterative approaches and provides a fast and computationally efficient solution. An interesting feature of the state-derivative feedback is that it in many cases gives feedback gains with smaller absolute values than traditional state feedback gains. The simulation results prove the feasibility and effectiveness of the proposed technique.

\section{References}

[1] Tuel, W. G.: On the transformation to (phase-variable) canonical form. IEEE Trans. on Automatic Control, AC-11, 1966, p. 607.

[2] Wonham, W. M.: On pole assignment in multi-input controllable linear systems. IEEE Trans. on Automatic Control, AC-12, 1967, p. 660-665.

[3] Luenberger, D. G.: Canonical forms for linear multivariable systems. IEEE Trans. on Automatic Control, AC-12, 1967, p. 290-292.

[4] Ackermann, J.: Der Entwurf linearer Regelungsysteme im Zustandraum. Regel. Tech. Proz.-Datenverarb., 1972, Vol. 7, p. 297-300.

[5] Wolowich, W. A.: Linear multivariable Systems. Springer Verlag, New York 1974.

[6] Kautsky, J., Nichols, N. K., Van Dooren, P.: Robust pole assignment in linear state feedback. Int. J. of Control, 1985, Vol. 41, p. 1129-1155.

[7] Lewis, F. L.: Applied Optimal Control and Estimation, Digital Design and Implimentation. Prentice-Hall and Texas Instruments, Englewood Cliffs, NJ., 1992.

[8] Valášek, M., Olgac, N.: An Efficient Pole Placement Technique For Linear Time-variant SISO Systems. IEE Control Theory Appl. Proc. D, 1995, Vol. 142, No. 5, p. 451-458.

[9] Valášek, M., Olgac, N.: Efficient Eigenvalue Assignments for General Linear MIMO Systems. Automatica, 1995, Vol. 31, No. 11, p. 1605-1617.

[10] Valášek, M., Olgac, N.: Pole Placement for Linear time-varying Non-lexicographically Fixed MIMO Systems. Automatica, 1999, Vol. 35, No. 11, p. 101-108.
[11] Preumont, A., Loix, N., Malaise, D., Lecrenier, O.: Active Damping of Optical Test Benches with Acceleration Feedback. Machine Vibration, 1993, Vol. 2, p. 119-124.

[12] Preumont, A.: Vibration Control of Active Structures. Kluwer, 1998.

[13] Bayon de Noyer, M. P., Hanagud, S. V.: Single Actuator And Multi-Mode Acceleration Feedback Control. Adaptive Structures and Material Systems, ASME, AD, 1997, Vol. 54, p. 227-235.

[14] Bayon de Noyer, M. P. Hanagud, S. V.: A Comparison of $\mathrm{H}_{2}$ Optimized Design and Cross-Over Point Design For Acceleration Feedback Control. Proceedings of $39^{\text {th }}$ AIAA/ASME/ ASCE/AHS, Structures, Structural Dynamics and Materials Conference, 1998, Vol. 4, p. 3250-3258.

[15] Olgac, N., Elmali, H., Hosek, M., Renzulli, M.: Active Vibration Control of Distributed Systems Using Delayed Resonator with Acceleration Feedback. Trans. of ASME Journal of Dynamic Systems, Measurement and Control, 1997, Vol. 119, p. 380.

[16] Kejval, J., Sika, Z., Valasek, M.: Active Vibration Suppression of a Machine. Proceedings of Interaction and Feedbacks '2000, UT AV CR, Praha, 2000, p. 75-80.

[17] Deur, J., Peric, N.: A Comparative Study of Servosystems with Acceleration Feedback. Proceedings of the $35^{\text {th }}$ IEEE Industry Applications Conference, Roma (Italy), 2000, Vol. 2, p. 1533-1540.

[18] Ellis, G.: Cures for Mechanical Resonance in Industrial Servo Systems. Proceedings of PCIM 2001 Conference, Nuremberg, 2001.

[19] Necsulescu, D. S., Ceru, M.: Nonlinear Control of Magnetic Bearing. Journal of Electrical Engineering, Vol. 2, 2002.

[20] Horn, R. A., Johnson, C. R.: Matrix Analysis. Cambridge University Press, Cambridge, 1986.

Prof. Ing. Michael Valášek, DrSc.

phone: +420224357361

e-mail: michael.valasek@fs.cvut.cz

Eng. Taha Helmy Sayed Abdelaziz, Ph.D.

e-mail: tahahelmy@yahoo.com

Department of Mechanics

Czech Technical University in Prague,

Faculty of Mechanical Engineering

Karlovo nám. 13

12135 Prague 2, Czech Republic 